\title{
Novel Pyoverdine Inhibitors Mitigate Pseudomonas aeruginosa Pathogenesis
}

\section{OPEN ACCESS}

Edited by:

Paolo Visca,

Università degli Studi Roma Tre, Italy

Reviewed by:

Pierre Cornelis,

Vrije Universiteit Brussel, Belgium

Rolf Kümmerli;

University of Zurich, Switzerland

${ }^{*}$ Correspondence:

Natalia V. Kirienko

kirienko@rice.edu

Specialty section:

This article was submitted to

Antimicrobials, Resistance and Chemotherapy,

a section of the journal

Frontiers in Microbiology

Received: 09 October 2018 Accepted: 20 December 2018

Published: 09 January 2019

Citation:

Kirienko $D R$, Kang $D$ and Kirienko NV (2019) Novel Pyoverdine Inhibitors Mitigate Pseudomonas

aeruginosa Pathogenesis.

Front. Microbiol. 9:3317.

doi: 10.3389/fmicb.2018.03317

\section{Daniel R. Kirienko, Donghoon Kang and Natalia V. Kirienko*}

Department of BioSciences, Rice University, Houston, TX, United States

Pseudomonas aeruginosa is a clinically important pathogen that causes a variety of infections, including urinary, respiratory, and other soft-tissue infections, particularly in hospitalized patients with immune defects, cystic fibrosis, or significant burns. Antimicrobial resistance is a substantial problem in $P$. aeruginosa treatment due to the inherent insensitivity of the pathogen to a wide variety of antimicrobial drugs and its rapid acquisition of additional resistance mechanisms. One strategy to circumvent this problem is the use of anti-virulent compounds to disrupt pathogenesis without directly compromising bacterial growth. One of the principle regulatory mechanisms for $P$. aeruginosa's virulence is the iron-scavenging siderophore pyoverdine, as it governs in-host acquisition of iron, promotes expression of multiple virulence factors, and is directly toxic. Some combination of these activities renders pyoverdine indispensable for pathogenesis in mammalian models. Here we report identification of a panel of novel small molecules that disrupt pyoverdine function. These molecules directly act on pyoverdine, rather than affecting its biosynthesis. The compounds reduce the pathogenic effect of pyoverdine and improve the survival of Caenorhabditis elegans when challenged with $P$. aeruginosa by disrupting only this single virulence factor. Finally, these compounds can synergize with conventional antimicrobials, forming a more effective treatment. These compounds may help to identify, or be modified to become, viable drug leads in their own right. Finally, they also serve as useful tool compounds to probe pyoverdine activity.

Keywords: Pseudomonas aeruginosa, antivirulence, siderophore, pyoverdine, Caenorhabditis elegans

\section{INTRODUCTION}

Despite advances in antimicrobial chemotherapy, multidrug resistant bacteria continue to cause life-threatening infections, especially in hospitals and with immunocompromised patients. Pseudomonas aeruginosa is a particularly pernicious pathogen as it possesses several innate defense mechanisms against antibiotics. For example, $P$. aeruginosa expresses four major efflux pumps (MexAB-OprM, MexCD-OprJ, MexEF-OprN, and MexXY-OprM) that effectively reduce intracellular drug concentrations, preventing the compounds from reaching intracellular doses required for effect (Lomovskaya et al., 2001). These pumps confer resistance to several classes of commonly used drugs, including $\beta$-lactams, fluoroquinolones, and aminoglycosides (Poole et al., 1993, 1996; Köhler et al., 1997; Aires et al., 1999). Chemically inhibiting these pumps significantly 
reduces the concentration of antimicrobial required for treatment (Lomovskaya et al., 2001). Unfortunately, P. aeruginosa also has several other mechanisms of resistance, including decreased membrane permeability and enzymes that modify or degrade antimicrobials (Kon and Rai, 2016). Drug targets are also prone to mutate to avoid antimicrobial sensitivity. In addition, $P$. aeruginosa produces robust biofilms that form a physical barrier between the pathogen and antimicrobial therapies (Mah and O'Toole, 2001; Anderson and O'Toole, 2008). P. aeruginosa readily acquires genetically encoded resistance determinants from other pathogens, further increasing resistance. The development and approval of new antimicrobials (especially new classes of antimicrobials) is typically a slower process than the development of resistance by the pathogen which also contributes to the increasing demand to at least supplement drug development efforts with novel treatment avenues for $P$. aeruginosa infections.

Perhaps the most promising route toward this goal is to target the virulence factors instead. Often, compromising these factors does not directly impinge on bacterial viability, which is predicted to reduce the pressure to evolve resistance. Virulence determinants come in a staggering array of forms, including toxins that directly damage the host (e.g., type III secretion effectors, pyocyanin, hemolysins, etc.) (Saliba et al., 2002; Cezairliyan et al., 2013; Managò et al., 2015; Dortet et al., 2018), proteins or co-factors that provide nutritional support for the pathogen (e.g., siderophores, carbohydrate permeases, etc.) (Minandri et al., 2016), or promote colonization (e.g., adherens, quorum-sensing, and biofilm formation). Smallmolecule inhibitors that target these systems mitigate bacterial pathogenicity, facilitating bacterial clearance by the host. Based on this idea, a number of antivirulence compounds have been proposed for $P$. aeruginosa, mostly targeting the pathogen's quorum-sensing systems and biofilm-forming capacity (Vandeputte et al., 2011; Tan et al., 2013; Imperi et al., 2014; Kang and Kirienko, 2017; Paczkowski et al., 2017; van Tilburg Bernardes et al., 2017; D’Angelo et al., 2018).

Although acute virulence determinants have generally received less attention in recent studies, the siderophore pyoverdine makes an attractive therapeutic target for several reasons. First, pyoverdine is essential for $P$. aeruginosa pathogenesis in various mammalian and invertebrate host models (Meyer et al., 1996; Takase et al., 2000; Imperi et al., 2013; Kirienko et al., 2013; Lopez-Medina et al., 2015; Minandri et al., 2016). Pyoverdine's exceptionally high affinity for ferric iron allows it to scavenge trace iron from the environment and also to abstract it from host iron-sequestering proteins such as transferrin and lactoferrin (Meyer et al., 1996; Xiao and Kisaalita, 1997). Second, pyoverdine can also hijack iron from other host sources, including mitochondria, which inflicts considerable damage on the host (Kirienko et al., 2013, 2015; Kang et al., 2018). Third, iron-bound pyoverdine (known as ferripyoverdine) functions as a signaling molecule that triggers the release of the alternate sigma factor PvdS from sequestration by the intermembrane FpvA/FpvR complex (Beare et al., 2003). Once released, PvdS promotes the expression of at least two secreted toxins (the translational inhibitor ToxA and the protease PrpL) and also its own biosynthetic machinery (Ochsner et al., 1996; Wilderman et al., 2001; Lamont et al., 2002). Fourth, the iron provided by pyoverdine is required for biofilm formation (Banin et al., 2005). Finally, and perhaps most importantly, fluoropyrimidines have been successfully used in several hosts to target pyoverdine, which has substantially reduced host mortality (Imperi et al., 2013; Costabile et al., 2016; Kirienko et al., 2016).

In this study, we describe the identification of a group of novel small molecules that compromise pyoverdine function without acting as conventional antimicrobials. We show that these compounds ameliorate pyoverdine-mediated damage, and that their effect is specifically due to their inhibition of pyoverdine function. These drugs rescue Caenorhabditis elegans at mid-micromolar concentrations and exhibit drug-like profiles. Promisingly, these compounds demonstrate synergy when combined with a conventional anti-Pseudomonal antibiotic. These compounds may serve as drug leads themselves or tool compounds for the identification of a future class of virulence inhibitors with clinical utility.

\section{MATERIALS AND METHODS}

\section{Bacterial and C. elegans Strains and Maintenance}

The strains used in this study can be found in Table 1. C. elegans strain SS104 [ $g l p-4(b n 2)]$ was maintained on nematode growth medium (NGM) seeded with Escherichia coli strain OP50 at $15^{\circ} \mathrm{C}$ (Stiernagle, 2006). P. fluorescens WCS365 (Geels and Schippers, 1983) was a gift from Dr. Cara Haney, and was selected as a high pyoverdine producer. The $P$. aeruginosa PA14pvdF mutant has a Mariner transposon inserted into the $p v d F$ locus, as verified by Sanger sequencing (Liberati et al., 2006). Enterococcus faecalis was a gift from Dr. Danielle Garsin.

\section{Caenorhabditis elegans - $P$. aeruginosa Assay}

The Liquid Killing assay was performed essentially as described (Anderson et al., 2018). In brief, $g l p-4(b n 2 t s)$ worms were synchronized by bleaching gravid adults and hatching in the absence of food at $15^{\circ} \mathrm{C}$. L1 larvae were dropped onto plates

TABLE 1 | Strains used in this study.

\begin{tabular}{lcc}
\hline Species & Strain & Reference \\
\hline C. elegans & glp-4(bn2) & Beanan and Strome, 1992 \\
E. coli & OP50 & Stiernagle, 2006 \\
P. aeruginosa & PA14 & Rahme et al., 1995 \\
P. aeruginosa & PA14 $\Delta$ pvdA & Shanks et al., 2006; Kuchma et al., 2007 \\
P. aeruginosa & PAO1 & Holloway et al., 1994 \\
P. aeruginosa & Boston 41501 & ATCC 27853 (Medeiros et al., 1971) \\
P. aeruginosa & KM 306 & ATCC 25010 (Yabuuchi and Ohyama, 1972) \\
P. aeruginosa & 6092 & ATCC 33360 (Liu et al., 1983) \\
P. fluorescens & WCS365 & Geels and Schippers, 1983 \\
E. faecalis & OG1RF & Garsin et al., 2001
\end{tabular}


containing standard E. coli OP50, and then transferred to the non-permissive temperature to induce sterility. At the young adult stage, they were washed from plates, and sorted into 384well plates containing $P$. aeruginosa. The bacteria were prepared by spreading a small amount of an overnight inoculum onto an SK media plate (Kirienko et al., 2014), and incubating the plate at $37^{\circ} \mathrm{C}$ for 1 day. Bacteria were then scraped from the plate and inoculated into LK media at a final $\mathrm{OD}_{600}$ of 0.03 .

For the assessment of potential synergistic interaction between antimicrobials and anti-virulents, C. elegans were incubated with $P$. aeruginosa strain PA14 for $24 \mathrm{~h}$, After that $200 \mu \mathrm{g} / \mathrm{mL}$ (final) of gentamicin with or without pyoverdine inhibitors $(100 \mu \mathrm{M})$ was added. Host survival was examined $24 \mathrm{~h}$ later using Sytox Orange staining as per standard Liquid Killing protocol.

\section{High-Throughput Screening Hits}

A high-content, high-throughput screen using the Liquid Killing assay was previously performed as described (Kirienko et al., 2016). Libraries with $>1000$ compounds screened are listed in Supplementary Table S1. Screening hits were purchased from varying companies through the chemical marketplace MolPort (Riga, Latvia). Small molecule purity (by HPLC) and identity (by mass spectrometry) was confirmed by vendors. When possible, compounds were sourced from more than one company to limit the possibility of misidentification. Analogous compounds were identified by searching chemical landscape using the Structure Search tools at MolPort.

\section{Production of Cell-Free, Pyoverdine-Rich Filtrate}

To produce pyoverdine-rich filtrate, $P$. aeruginosa or $P$. fluorescens strains were grown in modified M9 medium $[1 \%(\mathrm{w} / \mathrm{v})$ Difco $5 \times$ M9 salts, $11.3 \mathrm{~g} / \mathrm{L}$ casamino acids, $0.4 \%$ glucose, $1 \mathrm{mM}$ $\mathrm{CaCl} 2,1 \mathrm{mM} \mathrm{MgSO} 4]$ for $20-22 \mathrm{~h}$ at $28^{\circ} \mathrm{C}$ (P. fluorescens) or $37^{\circ} \mathrm{C}$ ( $P$. aeruginosa) with agitation. Subsequently, bacteria were pelleted by centrifugation at $10,000 \mathrm{~g}$ for $30 \mathrm{~min}$. Supernatants were sequentially filtered through $0.45,0.20$, and $0.20 \mu \mathrm{m}$ filters to remove residual bacteria. The resultant cell-free, pyoverdine-rich material was referred to as filtrate throughout the manuscript. All steps were performed using plastic containers.

\section{Pyoverdine Measurements}

A Cytation 5 multimode plate reader (BioTek Instruments, VT, United States) was used to assess quenching of pyoverdine by small molecules. To test pyoverdine fluorescence, $P$. aeruginosa strain PA14 was grown overnight in the presence of compounds, a single colony of $P$. aeruginosa strain PA14 was used to inoculate an overnight culture in LB, which was grown with agitation for $16 \mathrm{~h}$ at $37^{\circ} \mathrm{C}$. The resulting culture was diluted 1000 fold and inoculated into modified M9 medium (see below) containing compound, DMSO, or $\mathrm{FeCl}_{3}$ at $100 \mu \mathrm{M}$. Cultures were incubated at $37^{\circ} \mathrm{C}$ with shaking for $24 \mathrm{~h}$. The resulting cultures were centrifuged, and pyoverdine fluorescence in the media was determined spectrophotometrically with excitation $405 \mathrm{~nm}$ and emission $460 \mathrm{~nm}$. Similarly, the ability of the compounds to quench pyoverdine fluorescence in bacteria-free pyoverdine-rich filtrate was assayed by adding equal amounts of bacteria-free, pyoverdine-rich filtrate and modified M9 medium containing $100 \mu \mathrm{M}$ compound, DMSO, or $\mathrm{FeCl}_{3}$. Quenching was measured after a 5 min incubation at room temperature. All pyoverdine measurements were performed within the linear range of the Cytation 5, which was empirically determined to be between 1,000 and 20,000 arbitrary fluorescence units using serial dilutions of pyoverdine-rich cell-free filtrate.

\section{RNA Purification, NanoString, and qRT-PCR}

For NanoString (NanoString Technologies, WA, United States) studies, 2,000 worms were exposed to $100 \mu \mathrm{M}$ of LK11, LK31, LK31a, or ciclopirox olamine in S Basal media for $16 \mathrm{~h}$ in 6-well plates. Worms incubated in corresponding amount of DMSO for $16 \mathrm{~h}$ served as a normalization control. Afterward, worms were transferred into $15 \mathrm{~mL}$ conicals and washed twice with S Basal. Two biological replicates were tested for each condition. Gene expression was analyzed according to NanoString guidelines. For experiments involving quantification of expression of pyoverdine-dependent genes in the host, $g l p$ $4(b n 2)$ worms were exposed to pyoverdine for $16 \mathrm{~h}$ in the presence or absence of small molecules at $50 \mu \mathrm{M}$. RNA collection and qRT-PCR were performed as previously described (Kirienko et al., 2008). Fold-changes were calculated using a $\Delta \Delta \mathrm{Ct}$ method. Primer sequences are available upon request.

For assessing the effect of LK31 and LK31a on the expression of pyoverdine-dependent genes in $P$. aeruginosa, experiments were performed as follows: after $14 \mathrm{~h}$ growth in $6 \mathrm{~mL}$ of SK media supplemented with either DMSO or $100 \mu \mathrm{M}$ inhibitor, cells were collected via centrifugation. RNA was extracted and purified using TRIzol reagent (Invitrogen, CA, United States) according to the manufacturer's protocols with minor adjustments. To ensure cell lysis, cells resuspended in TRIzol reagent were freeze-cracked and vortexed prior to phase separation. Purified RNA was treated with DNase I (Thermo Fisher Scientific, MA, United States) to eliminate genomic DNA contamination. Reverse transcription was performed using random decamers and Retroscript Kit (Thermo Fisher Scientific, MA, United States). qRT-PCR was conducted using SYBR green AzuraQuant Fast Green Fastmix (Azura, MA, United States) in a CFX-96 real-time thermocycler (Bio-Rad, CA, United States). Fold-changes were calculated using a $\Delta \Delta \mathrm{Ct}$ method. Primer sequences are available upon request.

\section{Compounds' Effect Under Iron-Limiting Conditions}

WT $P$. aeruginosa PA14 or the PA14 $\Delta p v d A$ mutant were grown in 96-well plate in M9 media supplemented with casamino acids (BD Bacto, CA, United States) and with or without $6.25 \mu \mathrm{M}$ ciclopirox olamine (CPX). PA14 CPX samples were supplemented with either DMSO or pyoverdine 
inhibitors LK11, LK31, and LK31a at $100 \mu \mathrm{M}$. The 96-well plate was incubated in Cytation 5 multimode plate reader (BioTek, VT, United States) at $37^{\circ} \mathrm{C}$. $\mathrm{OD}_{600}$ readings were recorded every $30 \mathrm{~min}$ for $22.5 \mathrm{~h}$. Three biological replicates were performed.

\section{MIC, CFU, and Microscopy Assays}

To determine the minimum inhibitory concentration (MIC) of compounds for preventing bacterial growth, $P$. aeruginosa strain PA14 was grown in standard LB overnight and diluted 100,000fold in SK media. Compounds were two-fold serially diluted and mixed with equal volumes of diluted bacteria in 96-well plates. Plates were incubated at $37^{\circ} \mathrm{C}$ for $24 \mathrm{~h}$. Growth inhibition was visually scored on the basis of turbidity. Two wells were used per condition, and at least three biological replicates were performed. For quantification of colony-forming units (CFU), shaking cultures of E. coli strain OP50, P. aeruginosa strain PA14, or E. faecalis strain OG1RF were grown at $37^{\circ} \mathrm{C}$. At appropriate time points, aliquots were taken from each culture, serially diluted five-fold in S Basal, and plated onto LB agar. Colonies were counted under a dissecting microscope.

For assessing the impact of LK12 treatment on P. aeruginosa strain PA14, a strain carrying a plasmid encoding GFP was inoculated at an initial concentration of OD600 $=0.0001$ in the presence of either $250 \mu \mathrm{M}$ NK12 or DMSO alone $(1 \% \mathrm{v} / \mathrm{v})$. All cultures included propidium iodide at a final concentration of $40 \mu \mathrm{g} / \mathrm{mL}$. At 4 or $8 \mathrm{~h}$ after inoculation, bacteria were removed and imaged using a Zeiss M2 upright fluorescent microscope (Carl Zeiss, Germany). Experiments with Escherichia coli strain OP50 transformed with a GFP-encoding plasmid were performed in the same fashion.

\section{RESULTS}

\section{Selected Hits That Rescue C. elegans From $P$. aeruginosa Interfere With Pyoverdine Fluorescence}

We previously carried out a fragment-based, high-content, highthroughput phenotypic screen comprising over 86,000 wells (Kirienko et al., 2016). The goal was to identify compounds that increase $C$. elegans survival during exposure to $P$. aeruginosa strain PA14. This assay identified approximately 70 novel small molecules that passed initial and second-pass retesting that showed statistically significant rescue. Apparent pan-assay interfering compounds (Baell and Holloway, 2010; Baell and Walters, 2014) were removed, leaving 61 compounds, 54 of which were commercially available at the time of our initial retesting (Figure 1A). We have previously demonstrated that some of the hits acted by interfering with bacterial iron metabolism (Kirienko et al., 2013) or by inhibiting the production of pyoverdine (Kirienko et al., 2016). Since pyoverdine toxicity is the principal factor underlying host killing, we hypothesized that at least some of the remaining hits may act by blocking pyoverdine function.

In the past, we have observed a strong positive correlation between pyoverdine fluorescence and toxicity
(Kirienko et al., 2013; Kang et al., 2018). On this basis, we predicted that we would be able to identify compounds that compromise pyoverdine toxicity by measuring fluorescence in the presence of the compound. We tested the ability of each of the 54 commercially available hits to reduce pyoverdine fluorescence in growing cultures of $P$. aeruginosa PA14 (Figure 1B). We also added each of the commercially available compounds to pyoverdine-enriched, cell-free filtrates to verify that a reduction in fluorescence would be observed there as well, to rule out the possibility that the compounds were merely preventing pyoverdine production or were non-specifically compromising bacterial growth. Using these assays we identified four hits (LK10, LK11, LK12, and LK31) that quenched more than half of pyoverdine fluorescence (Figure 1C). These compounds also dramatically reduced pyoverdine fluorescence in $P$. aeruginosa PAO1, another clinically derived strain (Figure 1D).

Consistent with our predictions, the ability of the compounds to both rescue $C$. elegans (example shown in Figures 2A,B) and quench pyoverdine (Figure 2C) were dosage dependent. Interestingly, the four pyoverdine inhibitors represented diverse structural classes (Figure 2D). In an effort to better relate the structures and the activities of the compounds, we searched for commercially available analogs. We used a structurebased search tool to identify the closest commercially available compounds, and acquired up to three analogs for each (see Supplementary Figure S1 for structures). Each analog was solubilized in DMSO and tested for the ability to reduce pyoverdine fluorescence (Figures 3A,B). LK31a, which differs from its parent compound LK31 by the addition of a carbonitrile group (Figure 3C), showed significantly greater efficacy in this assay than its parent molecule. In contrast, LK31b had no discernible activity. None of the analogs selected for LK10, LK11, or LK12 exhibited anti-pyoverdine activity greater than their parent compounds.

Structurally, pyoverdine is comprised of two disparate parts. The chromophore, which is shared by all fluorescent Pseudomonads, consists of a relatively compact, heavily modified dihydroxyquinoline moiety. Attached to this is an oligopeptide chain that is produced by non-ribosomal protein synthesis machinery and introduces a variety of non-standard and $D$-conformation amino acids into the chain. The genes involved in biosynthesis of the oligopeptide side chain exhibit greater sequence variation than most regions of the $P$. aeruginosa genome. As a consequence, Pseudomonads produce pyoverdines that are sufficiently variable for use as a tool for taxonomic differentiation, a process called siderotyping (Meyer et al., 1998; Fuchs et al., 2001; Meyer et al., 2002). Early in the molecular study of pyoverdine, when it became apparent that pyoverdines had heterogeneous amino acid chains, they were designated as type I, type II, and type III (Meyer et al., 1997; De Vos et al., 2001). Since clinically relevant strains of $P$. aeruginosa are known to produce each of these types of pyoverdine, we obtained relevant strains of $P$. aeruginosa from the ATCC. We also tested compound efficacy against pyoverdine produced by $P$. fluorescens, which is likely to be even more variant from the type I producing strains we already tested (PAO1 and PA14). In each case, the compounds quenched pyoverdine fluorescence (Figure 4). 
A

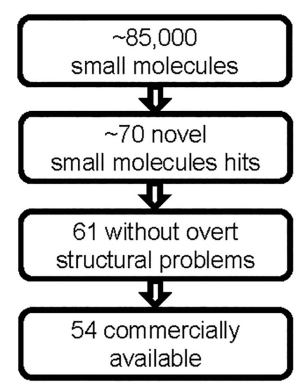

C

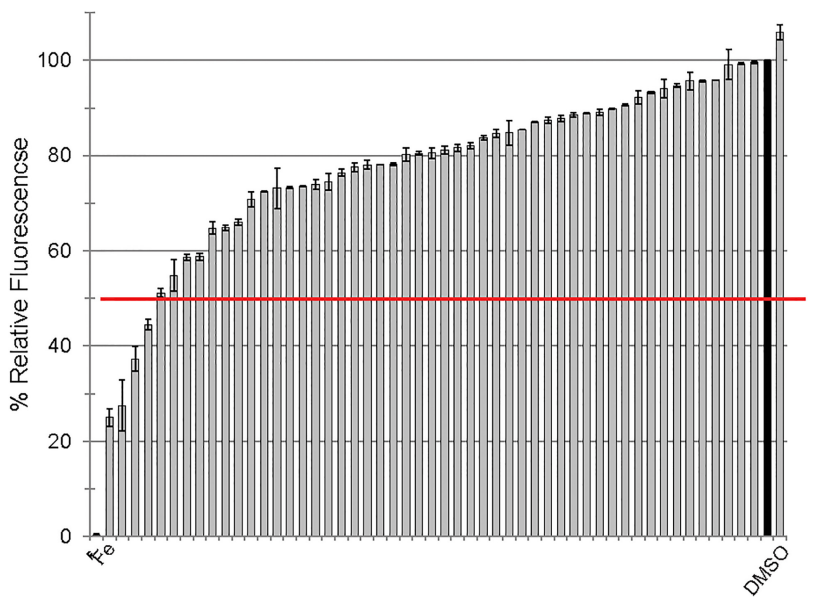

B

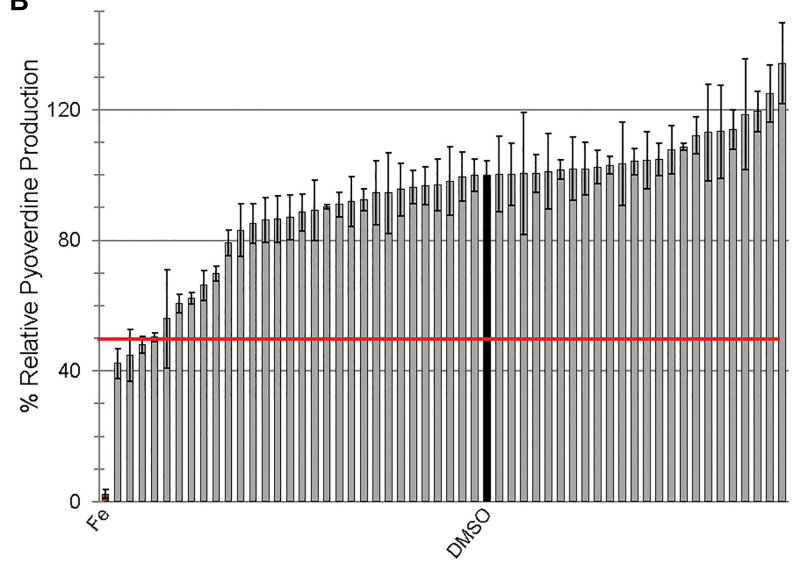

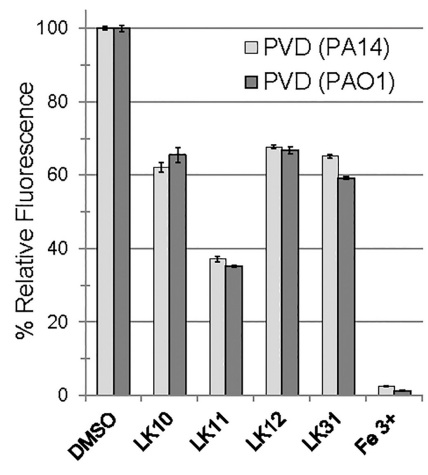

FIGURE 1 | Small molecules interfere with pyoverdine function. (A) Flow chart of screening summary for novel small molecules that rescue Caenorhabditis elegans from Pseudomonas aeruginosa. (B) Effect of small molecule hits on fluorescence of pyoverdine when bacteria were cultured $24 \mathrm{~h}$ in the presence of the compounds $(250 \mu \mathrm{M})$ or DMSO. (C) Fluorescence of pyoverdine in bacteria-free filtrate after a 5 min incubation with compound (250 $\mu$ M). (D) Selected small molecules are capable of quenching pyoverdine fluorescence from pyoverdine-rich filtrates produced by P. aeruginosa strain PA14 or PAO1. At least three biological replicates were performed for $\mathbf{B}-\mathbf{D}$. The red lines in $\mathbf{B}, \mathbf{C}$ correspond to $50 \%$ decrease in fluorescence.

\section{LK10 and LK12 Are Multifunctional Compounds}

Although LK10, LK11, LK12, and LK31 reduce pyoverdine fluorescence, we tested whether the compounds also act as antimicrobials by examining their ability to prevent bacterial growth. Compounds were serially diluted in 96-well plates and $P$. aeruginosa was added and grown overnight. We also tested several standard antimicrobials with efficacy against $P$. aeruginosa as controls. Compounds with MIC values similar to their effective rescuing concentrations (defined as the concentration required for statistically significant rescue in Liquid Killing) were considered to have antimicrobial activity. For comparison, the known antimicrobials we tested showed $\mathrm{MIC} / \mathrm{EC}$ ratios between 0.5 and 3 (Figure 5A). LK10 and LK12 also had MIC/EC ratios close to this range. On this basis, we have reclassified them as hybrid molecules, as they both inhibit $P$. aeruginosa growth and also quench pyoverdine fluorescence.

Since LK12 demonstrated the lowest MIC (suggesting the strongest antimicrobial effect), we further examined its antibacterial properties. We grew $P$. aeruginosa in the presence of varying concentrations of LK12 and observed a concentrationdependent bactericidal effect (Supplementary Figure S2A). Interestingly, this effect appeared transient, as bacterial growth quickly recovered and the titer of viable bacteria was high by 16 h. We tested whether the compound also affected E. coli or E. faecalis. We noted that, although the compound had a more pronounced effect on E. coli, it was still transient (Supplementary Figure S2B). We hypothesize that the difference in efficacy is related to the difference in efflux pump efficiency and expression between $P$. aeruginosa and E. coli. Compound treatment slowed E. faecalis growth, but there were no overt signs that it was bactericidal, even at twice the concentration used for the gramnegative bacteria. This may suggest that LK12 is more effective in blocking the growth and development of gram-negative bacteria.

To verify that the bacteria were dead, we also tested $P$. aeruginosa PA14 that constitutively express GFP. Bacteria were grown in the presence of the compound for $8 \mathrm{~h}$, and then washed and stained with propidium iodide. Propidium iodide is 


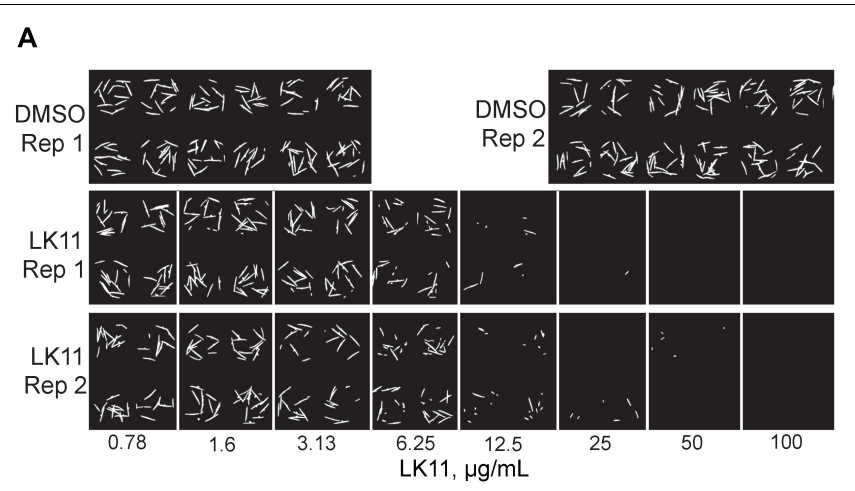

C

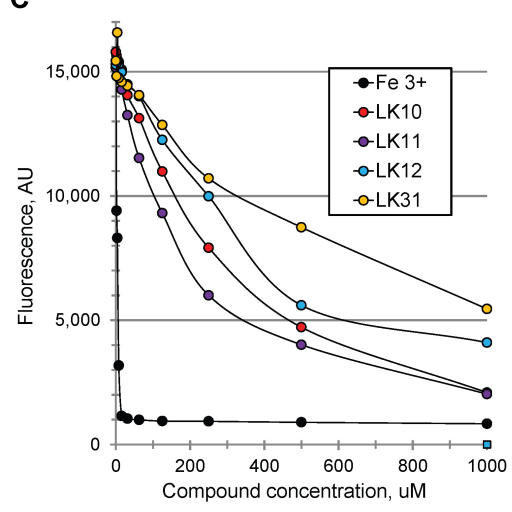

D<smiles>NNc1nc2ccccc2nc1S</smiles>

3-hydrazinylquinoxaline-2-thiol<smiles>N#Cc1c(N)[n+]([O-])c2ccccc2[n+]1[O-]</smiles>

LK12

3-amino1,4-dihydroxyquinoxaline-2-carbonitrile
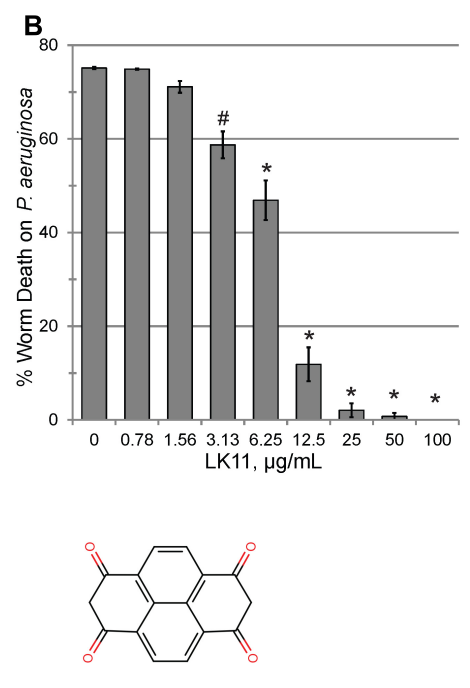

LK11

1,2,3,6,7,8-hexahydropyrene-1,3,6,8-tetrone

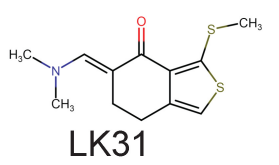

(5E)-5-[(dimethylamino)methylidene]-3-(methylsulfanyl)-4,5,6,7-tetrahydro-2-benzothiophen-4-one

FIGURE 2 | LK11 demonstrates dosage-dependent rescue. Representative images (A) and quantification (B) of $C$. elegans death after incubation with P. aeruginosa in the presence of varying concentrations of LK11. The cell-impermeable dye Sytox Orange was used to stain dead worms. Each well contains $\sim 18$ worms. At least three biological replicates were performed. (C) Pyoverdine-rich filtrate was incubated with various concentrations of pyoverdine inhibitors or iron (III) and fluorescence was measured after 5 minutes. (D) The structures and IUPAC names of of LK10, LK11, LK12, and LK31. $p$-values were calculated using Student's $t$-test, * $p<0.01$, ${ }^{\#} p<0.05$.

a cell-impermeant dye, and will stain only dead bacterial cells (Supplementary Figures S2C,D). After $8 \mathrm{~h}$, a large proportion of bacteria showed staining. Interestingly, we observed a very different phenomenon when we tried an analogous experiment using E. coli strain OP50. Instead of dividing properly, the morphology of $E$. coli cells changed to a longer, more filamentous state (Supplementary Figures S2E,F). This is consistent with a bacterial stress response to DNA damage (Suzuki et al., 1967; Walker and Pardee, 1968; Cushnie et al., 2016). Our findings are consistent with earlier reports about the capacity of LK12 to generate ROS and induce DNA damage (Ismail et al., 2010; Cheng et al., 2016).

\section{Anti-pyoverdines Have Drug-Like Properties}

We used the ability of LK11, LK31, and LK31a to quench pyoverdine fluorescence to determine their affinity for pyoverdine (Supplementary Table S2). Although the affinity of the compounds is relatively low, in the hundred micromolar range, this is common for 'very promising' hits from fragmentbased drug discovery methods (Murray and Rees, 2009). It is worth noting that no overt toxicity was seen when $C$. elegans was exposed to $128 \mu$ M LK11, LK31, or LK31a (Supplementary Figure S3). The other traits of the compounds generally conform to common drug design guidelines, such as Lipinski's Rule of Five (Lipinski et al., 2001) or The Rule of Three (Congreve et al., 2003; Table 2). These 'rules' have been empirically derived from successful drugs with known oral bioavailability and typically discriminate toward smaller, less reactive molecules. Consistent with this, all three have relatively low molecular weight (approximately 150-250 Da, Table 2). While not essential, low initial mass for leads is useful; drug development frequently involves adding functional groups that increase both affinity and mass. Based on these characteristics, LK11 and LK31/LK31a may serve as promising compounds for optimization. One intriguing possibility is that LK11 and LK31/LK31a could be combined into a single molecule with an even higher affinity if their binding sites are different and proximal to one another (Doak et al., 2016).

\section{Pyoverdine Inhibitors Behave as Bona Fide Anti-virulents}

Two factors suggested that LK11 and LK31 were acting as antivirulents. First, we were unable to use the compounds alone 


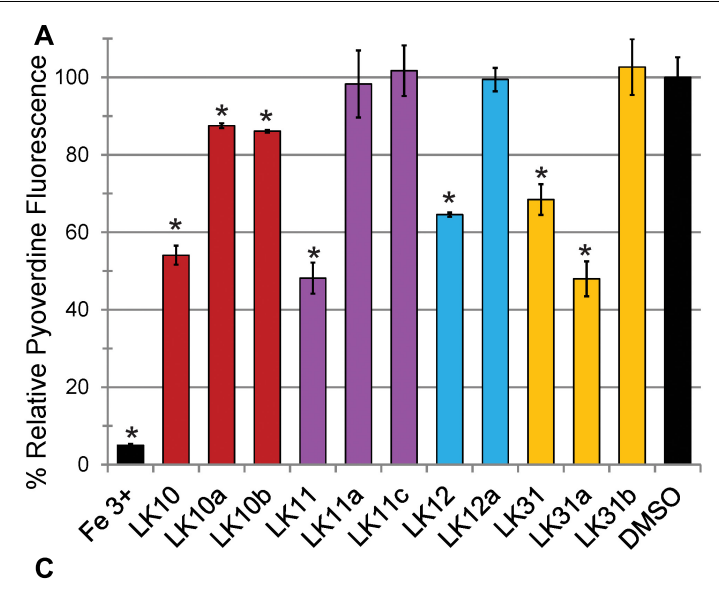

B

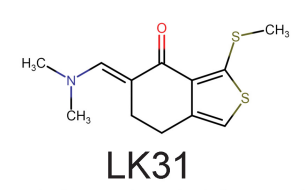

(5E)-5-[(dimethylamino)methylidene]-3-(methylsulfanyl)-4,5,6,7-tetrahydro-2-benzothiophen-4-one
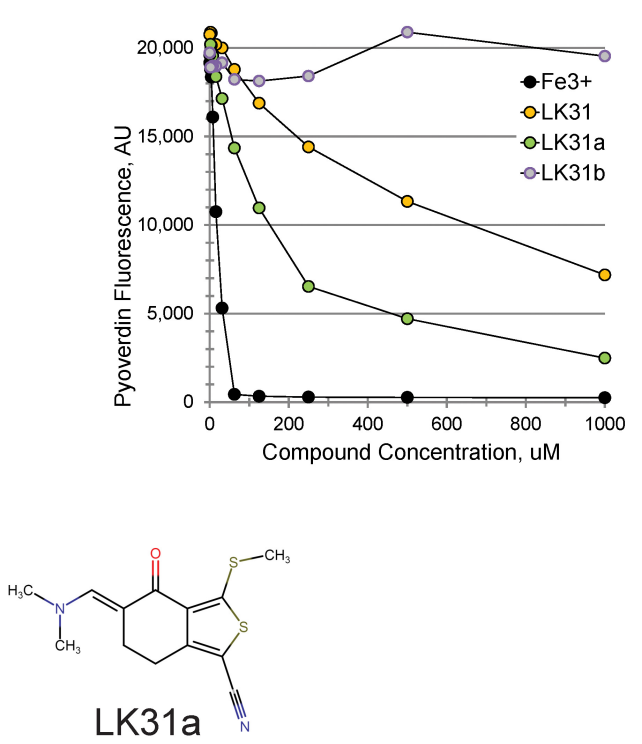

(5E)-5-[(dimethylamino)methylidene]-3-(methylsulfanyl)4-oxo-4,5,6,7-tetrahydro-2-bemzothiophene-1-carbonitrile

FIGURE 3 | Selected hits show dosage-dependent pyoverdine fluorescence quenching. (A,B) Fluorescence of bacteria-free, pyoverdine-rich filtrate after 5 min incubation with the compounds indicated at either fixed $(250 \mu \mathrm{M}, \mathbf{A})$ or varying (B) concentrations. DMSO. (C) Structural comparison of a parent compound (LK31) and an analog with improved efficiency (LK31a). At least three biological replicates were performed for A,B. $p$-values were calculated using Student’s $t$-test, $* p<0.01$.

to inhibit bacterial growth in standard MIC assays. Second, the MIC/EC ratios of LK11 and LK31 were at least an order of magnitude higher than the ratios for known antimicrobials. As pyoverdine is a large molecule requiring substantial cellular investment, disrupting or delaying bacterial growth significantly will delay pyoverdine biosynthesis. To rule out this potentially confounding issue, we carefully compared bacterial growth for cultures with various concentrations of LK11, LK31, LK31a, or DMSO alone. Rather than rely on spectroscopic measurements, which can be misleading, we serially diluted bacterial inocula and counted the number of viable bacterial colonies. Bacterial growth kinetics were unaffected by the presence of LK11, LK31, or LK31a in two different growth media (Figures 5B,C).

Under iron-limiting conditions pyoverdine becomes essential for bacterial survival and growth. For this reason, we anticipated that the compounds may compromise bacterial growth under these conditions. First, we compared the growth of wildtype $P$. aeruginosa PA14 and $\Delta p v d A$ pyoverdine production mutant in M9 media supplemented with casamino acids. In the absence of an exogenous iron chelator, growth of the strains was indistinguishable (Figure 6). In contrast, the addition of $6.25 \mu \mathrm{M}$ ciclopirox olamine (CPX) caused a small lag in wild-type development but compromised the growth of the $\Delta p v d A$ mutant, indicating that these conditions require pyoverdine for growth. Adding LK11, LK31, or LK31a to wild-type P. aeruginosa growing in the presence of CPX phenocopied the $\Delta p v d A$ defect, providing another piece of evidence to support that the compounds are compromising pyoverdine function.
Although these data substantially demonstrated that the pyoverdine inhibitors were compromising pyoverdine fluorescence and that they were not functioning by preventing bacterial growth under iron replete conditions, we also wanted to eliminate the possibility that their effect was due to the compounds exerting some uncharacterized effect on the host. For example, previous reports have shown that small molecules can trigger host defenses and extend C. elegans survival, even if they are toxic and will eventually kill the host as well (PukkilaWorley et al., 2012). To test this, we used NanoString technology to assess the mRNA levels of 115 genes involved in C. elegans immune and stress response pathways. Gene expression was normalized to three housekeeping genes; (see Supplementary Table S3 for the list of genes and fold-changes). mRNA was purified from C. elegans treated with either LK11, LK31, LK31a, or DMSO (as a control) in the absence of $P$. aeruginosa or pyoverdine. LK11 had minimal effect on the host, with only 4 genes (3.5\%) showing upregulation between 3 - and 10-fold. LK31 and LK31a each upregulated less than 10\% of the genes surveilled (and generally upregulated the same genes, which is consistent with their structural similarity) (Table 3). In contrast, $\sim 34 \%$ of the genes were upregulated in C. elegans exposed to the iron chelator ciclopirox olamine.

The screening assay used automatic scoring, and host death was inferred on the basis of staining with the cell impermeant dye Sytox Orange. Therefore, we also ruled out the possibility that the compounds were somehow preventing the dye from staining dead worms (or that the anti-pyoverdine compounds were 
A

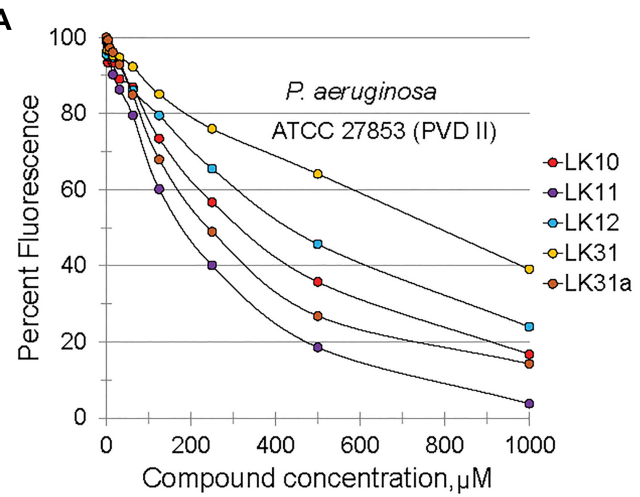

C

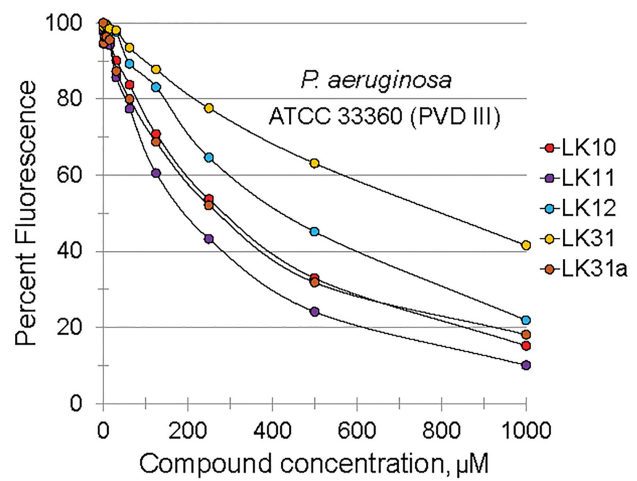

B

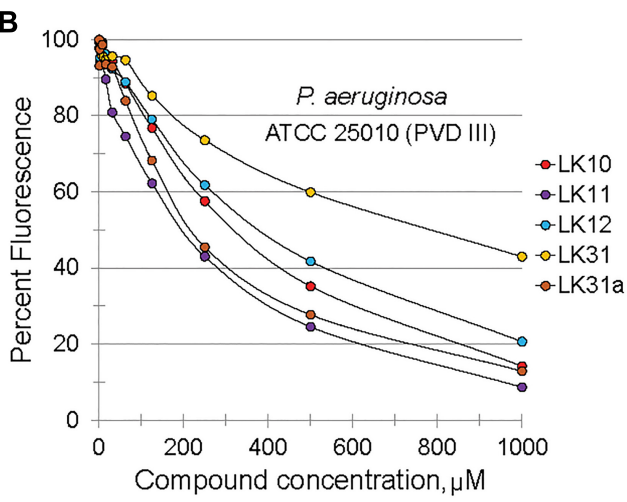

D

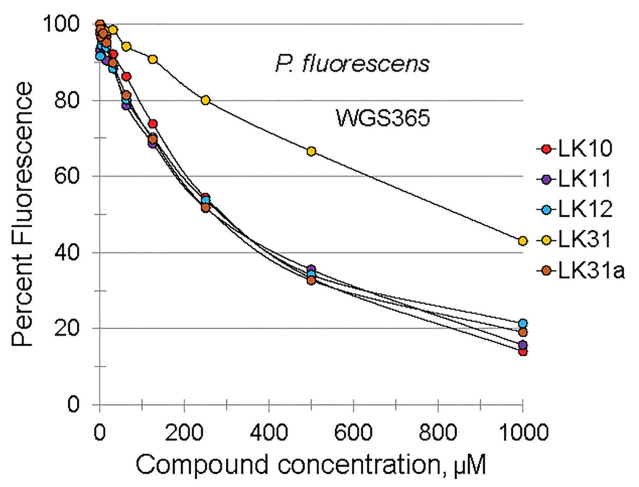

FIGURE 4 | Selected hits inhibit fluorescence of structurally diverse pyoverdines. (A-D) Fluorescence of bacteria-free, pyoverdine-rich filtrate after 5 min incubation with compounds at varying concentrations or DMSO. Filtrates were produced from the indicated strains. At least two biological replicates were performed.

interfering with the fluorescence of the dye) by staining heatkilled worms in the presence of the compounds (Supplementary Figure S4). As expected, staining was unaffected.

\section{Anti-pyoverdine Compounds Specifically Minimize Pyoverdine Toxicity}

We confirmed that LK11, LK31, and LK31a reduced bacterial virulence in C. elegans. As expected, all three compounds showed a strong, dose-dependent impact, limiting pathogenesis in the mid-micromolar range (Figure 7A). Based on our observations, these compounds are most likely functioning largely or entirely by limiting pyoverdine-mediated virulence. To further test this assertion, we measured the expression of pyoverdineresponsive genes (Kang et al., 2018) in C. elegans exposed to $P$. aeruginosa in the presence of LK11, LK31, LK31a, or DMSO. Upregulation of these genes was significantly diminished (Figure 7B) indicating that the toxicity of pyoverdine has been substantially blunted.

As noted above, iron-bound pyoverdine releases the alternative sigma factor $\mathrm{PvdS}$ from its sequestration at the plasma membrane, promoting the expression of several products, including itself, ToxA, and the proteast PrpL (Ochsner et al., 1996; Wilderman et al., 2001; Lamont et al., 2002). If the compounds in question are compromising pyoverdine function, we would expect transcription of these genes to be repressed in their presence. To test this, we assessed expression of PvdS-dependent genes (including $p v d S$, $p v d A, p v d E, p v d F$, toxA, and $p r p L)$ in $P$. aeruginosa PA14 grown in the presence of either DMSO, LK31, or LK31a, and observed that the compounds reduced their expression (Supplementary Figure S5). We also examined the expression of genes involved in pyochelin biosynthesis as a control. These genes were unaffected by treatment, as would be expected.

Although the anti-pyoverdine compounds LK11, LK31, and LK31a clearly quench pyoverdine fluorescence, reduce pyoverdine toxicity, and significantly decrease the pathogenesis of $P$. aeruginosa, it is formally possible that these phenomena are unrelated and that virulence attenuation is due to some other mechanism. The most straightforward way to test this is to assay whether the compounds mitigate $P$. aeruginosamediated virulence caused by a pyoverdine biosynthesis mutant. Pyoverdine mutants produce little to no pyoverdine and exhibit substantially reduced rates of killing C. elegans (Kirienko et al., 2013). We evaluated the ability of the $P$. aeruginosa PA14pvdF mutant strain to kill C. elegans when LK11, LK31, or LK31a are added. With the exception of the highest concentration tested (7- to 10-fold higher than the calculated EC), the compounds 
A

\begin{tabular}{|l|c|c|c|}
\hline & MIC & EC & MIC/EC \\
\hline Ciprofloxacin & 0.42 & 0.32 & 1.33 \\
\hline Gentamicin & 13.13 & 22.97 & 0.57 \\
\hline Polymyxin B & 1.12 & 0.26 & 2.72 \\
\hline LK10 & 130.2 & 65.1 & 2.00 \\
\hline LK11 & $>400$ & 11.47 & $>34.87$ \\
\hline LK12 & 82.6 & 61.8 & 1.34 \\
\hline LK31 & $>400$ & 18.93 & $>21.13$ \\
\hline LK31a & $>400$ & 12.47 & $>32.01$ \\
\hline
\end{tabular}

B

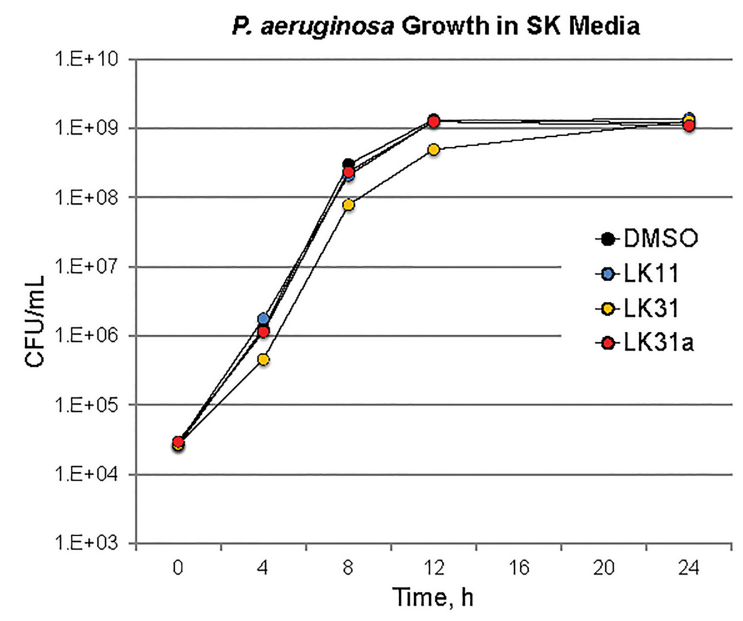

C

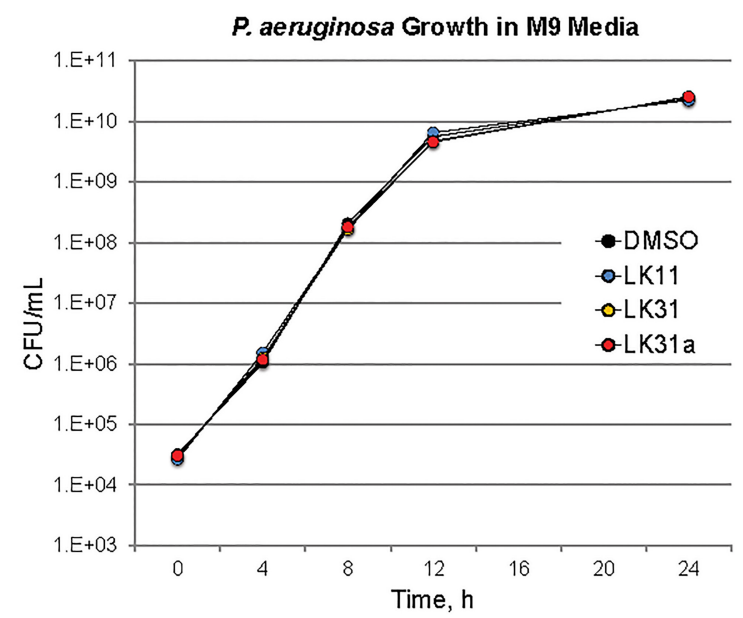

FIGURE 5 | A subset of pyoverdine inhibitors behave as anti-virulents.

(A) MICs, effective concentrations (EC, defined as the minimum concentration required for statistically significant amelioration of killing), and the ratios of MIC to $E C$ are shown for a panel of classical antimicrobials, the antivirulents $L K 11$, LK31, and LK31a, and the bifunctional compounds LK10 and LK12. (B,C) Colony-forming unit counts for $P$. aeruginosa grown in the presence of DMSO or $100 \mu \mathrm{M}$ of LK11, LK31, and LK31a in nutrient-poor SK media (B) or more nutritious M9 media (C). At least three biological replicates were performed for each experiment.
TABLE 2 | Chemical properties of pyoverdine inhibitors.

\begin{tabular}{lcccccc}
\hline Molecule & MW, Da & LogP & Polar area & $\begin{array}{c}\text { H Bond } \\
\text { donor }\end{array}$ & $\begin{array}{c}\text { H Bond } \\
\text { acceptor }\end{array}$ & $\begin{array}{c}\text { Rotatable } \\
\text { bonds }\end{array}$ \\
\hline LK11 & 264.2 & 2.1 & 68.3 & 0 & 4 & 0 \\
LK31 & 253.4 & 2.9 & 73.8 & 0 & 4 & 2 \\
LK31a & 278.4 & 2.9 & 97.6 & 0 & 5 & 2 \\
Goal & $<500$ & $-0.4 \ldots 5.6$ & $<1$ & $<5$ & $<10$ & $<10$ \\
\hline
\end{tabular}

had little to no effect on the ability of the mutant strain to kill C. elegans (Figure 7C).

\section{Anti-pyoverdine Compounds Synergize With Antibacterial Agents}

In addition to serving as monotherapies, antivirulent drugs also have the potential to be included in therapeutic "cocktails" of multiple drugs that have synergistic activities. For example, the combination of pyoverdine inhibitors (to mitigate siderophore toxicity) and bacteriostatic or bactericidal drugs (to limit bacterial growth) is likely to be more effective than either drug alone. This is particularly due to pyoverdine's ability to support other virulence factors, such as biofilms (reviewed in Kang and Kirienko, 2018). We predicted that the combination of an antipyoverdine like LK11, LK31, or LK31a with gentamicin would result in increased treatment efficiency in Liquid Killing when compared to antibiotic alone. To test this, we exposed worms to $P$. aeruginosa for $24 \mathrm{~h}$ and then added either antibiotic alone or antibiotic and an anti-pyoverdine compound. All three hit compounds showed a synergistic effect compared to antibiotic alone (Figure 8).

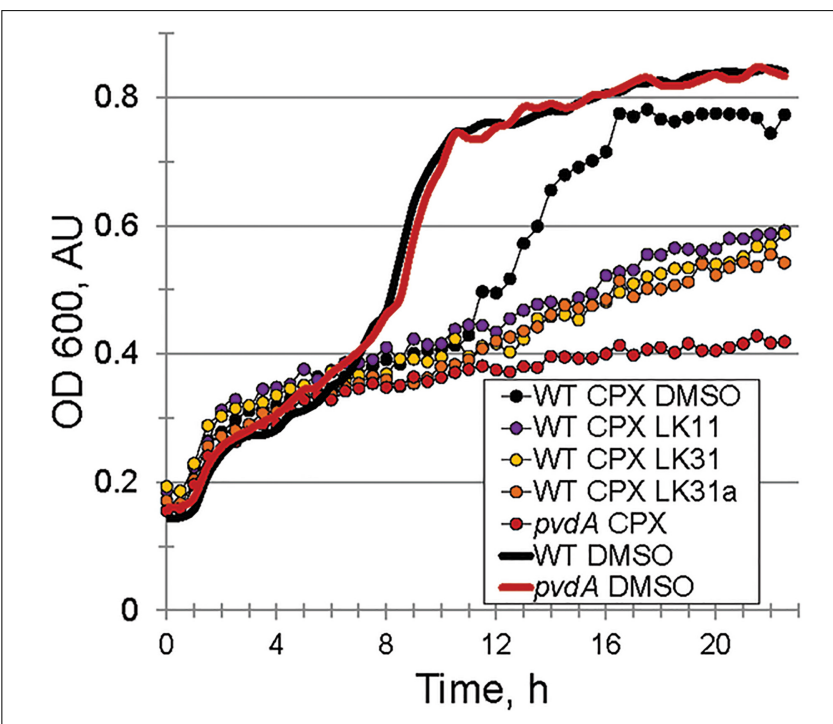

FIGURE 6 | Pyoverdine inhibitors disrupt bacterial growth under iron-limiting condition. Growth curves of WT P. aeruginosa PA14 and PA14pvdA. Iron limitation was induced by the presence of $6.5 \mu \mathrm{M}$ ciclopirox olamine (CPX), an iron (III) chelator. DMSO or compounds were added at $100 \mu \mathrm{M}$. Three biological replicates were performed. 
TABLE 3 | Pyoverdine inhibitors do not trigger overt host defense response.

\begin{tabular}{lcccc}
\hline & LK11 & LK31 & LK31a & CPX \\
\hline$>3,<10$ & 4 & 8 & 8 & 24 \\
$>10,<50$ & 0 & 2 & 2 & 10 \\
$>50$ & 0 & 0 & 0 & 5 \\
Sum up & 4 & 10 & 10 & 39 \\
$\%$ Up & 3.5 & 8.7 & 8.7 & 33.9
\end{tabular}

CPX - ciclopirox olamine, iron chelator. The number shown represents the number of genes in each category. Sum up - total number of upregulated genes. \% Up percentage of upregulated genes (out of 115 total).

\section{DISCUSSION}

\section{Possible Mechanisms for Anti-pyoverdine Compounds}

We observed that anti-pyoverdine compounds can quench the fluorescence of pyoverdine in cell-free filtrates, limit the expression of pyoverdine-dependent genes, and improve C. elegans survival after exposure to $P$. aeruginosa strain PA14. It is worth noting that, although they are effective at conferring resistance to pyoverdine, they do not completely abolish its activity, and are unlikely to compromise pyoverdine biosynthesis. This distinction is important for two reasons. First, non-vaccine treatments for bacterial pathogens need to be effective when used after infection. Second, $P$. aeruginosa recycles and reuses pyoverdine (Greenwald et al., 2007; Imperi et al., 2009). After import, oxidoreductases act upon ferripyoverdine to reduce iron (III) to iron (II), lowering the siderophore's affinity for the metal. This facilitates the non-destructive removal of iron. Afterward, iron-free pyoverdine is exported to continue its activities, including inflicting additional damage and promoting toxin expression. For these reasons, preventing the biosynthesis of pyoverdine is less desirable than compromising its activity.

It remains an open question how LK11, LK31, and LK31a interact with pyoverdine. The simplest explanation for our observations is that these compounds bind to the conserved dihydroxyquinoline chromophore. This would explain the ability of the molecules to quench pyoverdine fluorescence, which is dependent upon this region of the molecule. It would also explain how the compounds can effectively quench the fluorescence of such disparate pyoverdines. Unfortunately, it is difficult to rule out the possibility that the compounds are binding the peptide sidechains. The short length of these chains (generally between 8 and 15 amino acids) may limit the biophysical interactions that generally drive protein folding. As such, these peptide sequences could exhibit conformational similarities despite differences in primary sequence.

These compounds also restrict the production of pyoverdinedependent virulence factors, including exotoxin A, the protease PrpL, and pyoverdine itself. Although we have not formally demonstrated that the pyoverdine-compound complex is incapable of binding to the FpvA receptor or that it prevents the release of PvdS from subcellular sequestration, the qRT-PCR data indicate that PvdS-mediated transcription is significantly reduced after compound treatment (Supplementary Figure S5).

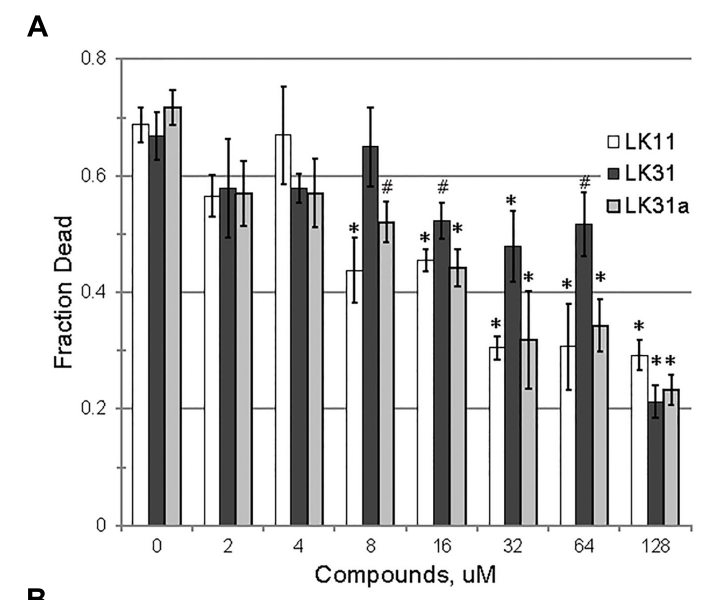

B

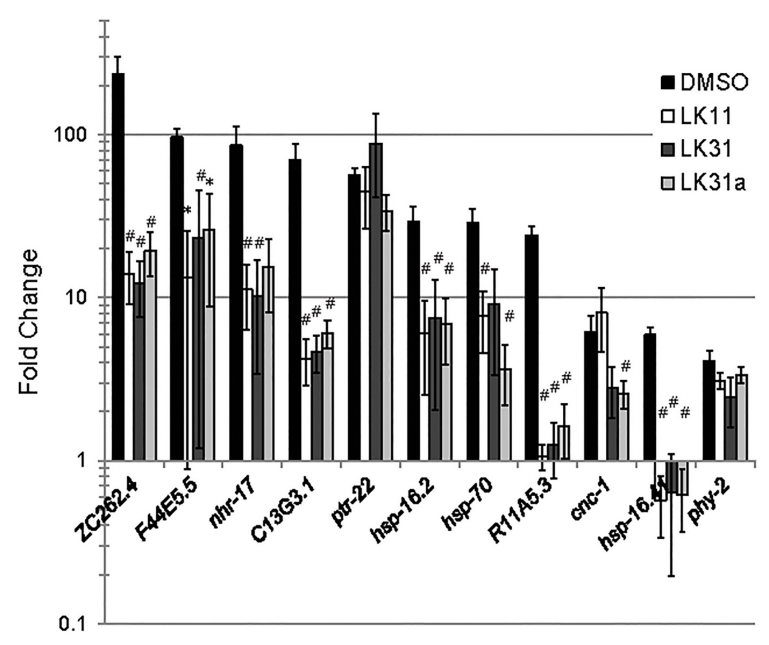

C

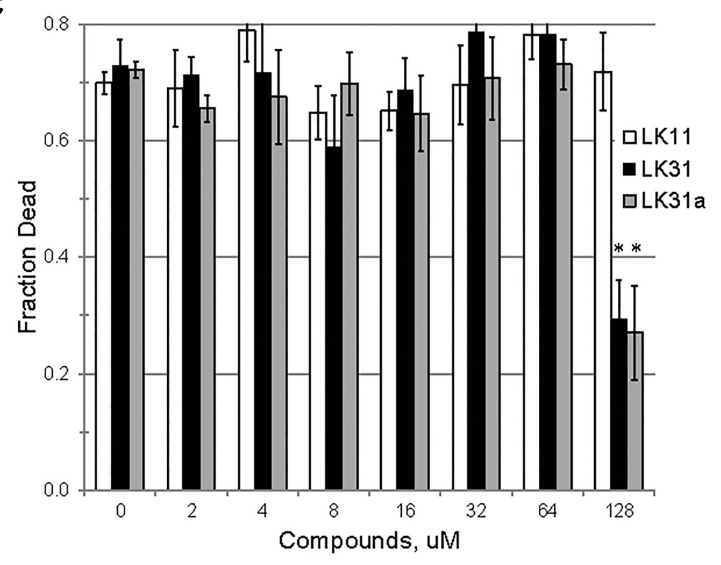

FIGURE 7 | LK11, LK31, and LK31a inhibit pyoverdine-mediated pathology. (A) Liquid Killing of $C$. elegans was assayed in the presence of varying doses of LK11, LK31, and LK31a. (B) qRT-PCR showing expression of a panel of genes upregulated by pyoverdine exposure during Liquid Killing of $C$. elegans treated with LK11, LK31, or LK31a $(100 \mu \mathrm{M})$. (C) Liquid Killing of C. elegans exposed to $P$. aeruginosa strain PA14pvdF, which is defective in pyoverdine biosynthesis, and treated with LK11, LK31, or LK31a at the concentrations indicated. At least three biological replicates were performed for A-C. $p$-values were calculated using Student's $t$-test, ${ }^{*} p<0.01,{ }^{\#} p<0.05$. 


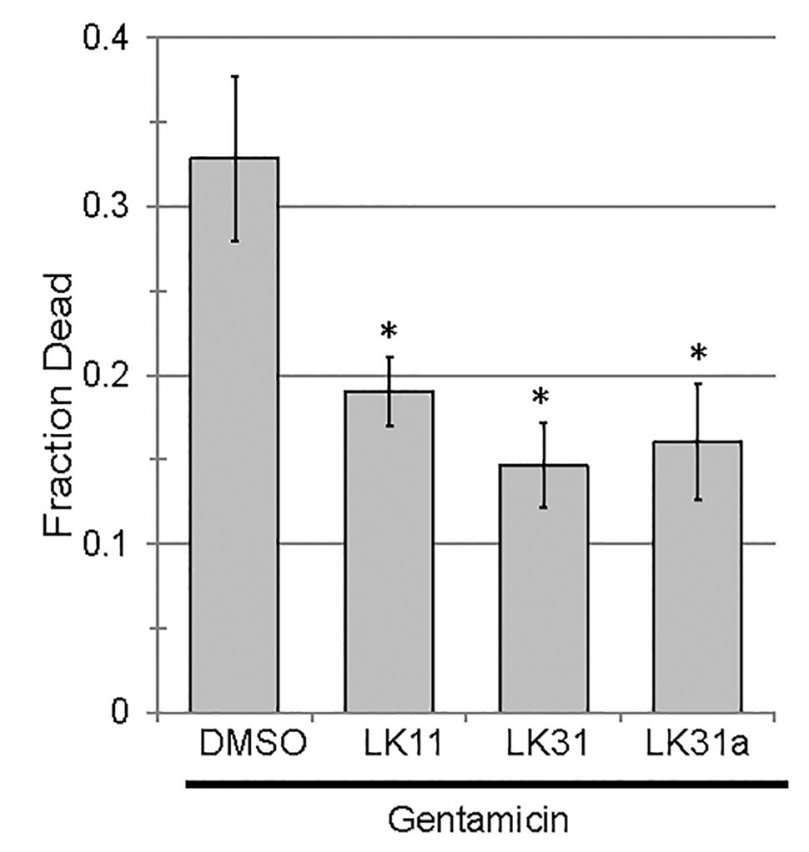

FIGURE 8 | Pyoverdine inhibitors synergize with antimicrobial gentamicin. Liquid Killing of $C$. elegans exposed to $P$. aeruginosa for $24 \mathrm{~h}$ and subsequently treated with either gentamicin alone or gentamicin in combination with an antivirulent $(100 \mu \mathrm{M})$. At least three biological replicates were performed. $p$-values were calculated using Student's $t$-test, ${ }^{*} p<0.01$.

The most parsimonious explanation for this is that the compounds have prevented one or more of these feed-forward steps. This demonstrates the power of anti-virulents and validates pyoverdine as a target, since even these non-optimized hits show considerable potential to alleviate virulence in this model. The next step will be to test their ability to limit pathogenesis in a more complex host.

The other matter that remains to be resolved is whether the compounds preclude pyoverdine from binding iron. As demonstrated with ciclopirox olamine, these compounds are likely to transition from anti-virulents to antimicrobials under strict iron limitation, which is likely to be the case in most hosts, including humans. Anti-virulents are very desirable because they are anticipated to reduce the evolutionary pressure to acquire resistance. As such, it is undesirable for the pathogen to transition to a state where they act as antimicrobials. Ultimately, however, this difference may be somewhat academic. Virulence is a complex trait, associated with the expression of a wide variety of genes and the establishment of a specific homeostatic state in the pathogen. Using small molecules to meddle with this state could easily disrupt the pathogen's ability to survive or reproduce, with unpredictable consequences that are likely to depend upon the nature of the resistance that arises (Allen et al., 2014; Rezzoagli et al., 2018).

One possible outcome is that disrupting pyoverdine would stimulate increased production of alternative iron acquisition systems, like pyochelin. It is difficult to predict what effect this would have on virulence; typically, pyochelin is generally reported to be dispensable for virulence in mammalian systems, especially when pyoverdine biosynthesis is intact (Takase et al., 2000; Minandri et al., 2016).

A less likely, but not disproven, possibility is that the compounds are preventing pyoverdine from carrying out its other activities without compromising its ability to bind iron. For example, previous reports have shown that pyoverdine inflicts considerable damage on mitochondria and activates mitochondrial surveillance pathways (Kirienko et al., 2015; Tjahjono and Kirienko, 2017; Kang et al., 2018). This almost certainly requires that the siderophore traverses the plasma membrane, both to directly acquire the iron that it removes as well as to bring it back to the pathogen. This is consistent with prior data from our lab that showed the pyoverdine removed iron from the host (Kang et al., 2018). This directly contrasts with another iron chelator, phenanthroline, which caused similar damage by chelating iron, but did not leave C. elegans. If the compounds are interfering with the ability of the siderophore to enter or exit host cells, virulence would be compromised in Liquid Killing, but iron acquisition in normal media would probably be unaffected.

\section{Other Methods to Target Pyoverdine}

The contributions of pyoverdine cytotoxicity to mammalian pathology remain important and unresolved, but pyoverdine clearly represents a valuable therapeutic target. To date, several approaches have been taken to target the pyoverdine siderophore system. Based on work by the Quax Lab (Nadal Jimenez et al., 2010), several groups have targeted PvdQ, which is required for pyoverdine maturation. The Gulick Lab carried out a highthroughput screen to identify small molecule inhibitors, and found a biaryl nitrile inhibitor of PvdQ (Drake and Gulick, 2011; Wurst et al., 2014). Other hits were identified by the Schreiber labs (Theriault et al., 2010) and a boronated alkyl chain by the Fast lab (Clevenger et al., 2013).

Interestingly, the biaryl nitrile inhibitor appears to synergize with a second class of pyoverdine inhibitors, fluoropyrimidines (Wurst et al., 2014). These compounds, including 5-fluorocytosine (5-FC) and 5-fluorouridine (5-FU) have been shown to compromise pyoverdine biosynthesis (Imperi et al., 2013; Costabile et al., 2016; Kirienko et al., 2016). Normally used as an antifungal, 5-FC has proven to be an effective method to inhibit $p v d S$ expression (Imperi et al., 2013). This sigma factor is necessary for expression of the biosynthetic machinery that produces pyoverdine (Lamont et al., 2002). Suppression of $p v d S$ in this fashion is sufficient to mediate rescue in C. elegans and in mice (Imperi et al., 2013; Kirienko et al., 2016). Interestingly, the mechanism of action remains unknown, but appears to require conversion of 5-FC to the well-known chemotherapeutic 5-fluorouracil (Imperi et al., 2013). From there, the drug is further metabolized to 5-fluorouridine (Kirienko et al., 2016). It remains unclear how this latter disrupts pyoverdine biosynthesis. Nevertheless, while fluoropyrimidines may serve as drugs of last resort for patients infected with pandrug-resistant $P$. aeruginosa, their profound cytotoxicity would appear to limit the potential of these therapies. 
Another widely explored approach is to directly target pyoverdine by adding gallium (III) to the system to compete with $\mathrm{Fe}^{3+}$. Although the ionic radii of $\mathrm{Ga}^{3+}$ and $\mathrm{Fe}^{3+}$ are nearly identical, and most proteins that use $\mathrm{Fe}^{3+}$ as a cofactor can incorporate $\mathrm{Ga}^{3+}$, gallium is redox inactive under biological conditions. Since the removal of $\mathrm{Fe}^{3+}$ from pyoverdine requires its reduction to $\mathrm{Fe}^{2+}, \mathrm{Ga}^{3+}$ becomes irreversibly bound to pyoverdine. Tests in animal models show that gallium can disrupt $P$. aeruginosa growth and inhibit biofilm formation (Kaneko et al., 2007; Banin et al., 2008; DeLeon et al., 2009). Unfortunately, $\mathrm{Ga}^{3+}$ has proven less effective in human serum, as elegant work by Paolo Visca's group demonstrated that the combination of $P$. aeruginosa proteases and pyoverdine's capacity for removing iron from host proteins facilitated pathogen growth, even in the presence of maximally achievable concentrations of $\mathrm{Ga}^{3+}$ (Bonchi et al., 2015). Although it remains an open question whether gallium will be more effective in human pulmonary environments, the promise of the early results is tempered by these findings. Some available evidence also suggests that gallium-bound pyoverdine still triggers the production of most pyoverdine-dependent virulence factors (Garcia-Contreras et al., 2014), although others have not seen this effect (Rezzoagli et al., 2018). Several other concerns, such as the immunosuppressive effect of $\mathrm{Ga}^{3+}$ and the difficulty of effectively using gallium as a treatment after the establishment of an infection (as opposed to pre-treatment or simultaneous treatment, as was used in most model assays) have also been expressed (Minandri et al., 2014).

Although none of the antivirulents described for P. aeruginosa are ready for clinical use, it is quite clear that new treatments, especially those that are not conventional antimicrobials, are urgently needed. Pyoverdine represents an invaluable target, due to its direct toxicity, position at the crux of nutrient acquisition, and regulation of virulence factors. Accordingly, it is likely that it will be an important target for chemical intervention. The antivirulents in this paper demonstrate proof of principle for

\section{REFERENCES}

Aires, J. R., Köhler, T., Nikaido, H., and Plésiat, P. (1999). Involvement of an active efflux system in the natural resistance of Pseudomonas aeruginosa to aminoglycosides. Antimicrob. Agents Chemother. 43, 2624-2628. doi: 10.1128/ AAC.43.11.2624

Allen, R. C., Popat, R., Diggle, S. P., and Brown, S. P. (2014). Targeting virulence: can we make evolution-proof drugs? Nat. Rev. Microbiol. 12, 300-308. doi: $10.1038 /$ nrmicro3232

Anderson, G. G., and O'Toole, G. A. (2008). Innate and induced resistance mechanisms of bacterial biofilms. Curr. Top. Microbiol. Immunol. 322, 85-105. doi: 10.1007/978-3-540-75418-3_5

Anderson, Q. L., Revtovich, A. V., and Kirienko, N. V. (2018). A high-throughput, high-content, liquid-based C. elegans pathosystem. J. Vis. Exp. 137:e58068. doi: $10.3791 / 58068$

Baell, J., and Walters, M. A. (2014). Chemistry: chemical con artists foil drug discovery. Nature 513, 481-483. doi: 10.1038/513481a

Baell, J. B., and Holloway, G. A. (2010). New substructure filters for removal of pan assay interference compounds (PAINS) from screening libraries and for their exclusion in bioassays. J. Med. Chem. 53, 2719-2740. doi: 10.1021/jm901137j

Banin, E., Lozinski, A., Brady, K. M., Berenshtein, E., Butterfield, P. W., Moshe, M., et al. (2008). The potential of desferrioxamine-gallium as an anti-Pseudomonas targeting pyoverdine's function, rather than its synthesis. They also represent promising fragments for lead generation and will serve as useful tool compounds for new P. aeruginosa discovery.

\section{AUTHOR CONTRIBUTIONS}

All authors designed and performed the experiments, analyzed the results, edited the manuscript, and were aware of this submission. NK supervised the project. DKi wrote the first draft. NK acquired funding.

\section{FUNDING}

This study was supported by the National Institute of Health grants K22AI110552 and R35GM129294, by the Welch Foundation grant C-1930, and the Cancer Prevention and Research Institute of Texas grant RR150044 awarded to NK. The funders had no role in study design, data collection and analysis, decision to publish, or preparation of the manuscript.

\section{ACKNOWLEDGMENTS}

Pseudomonas fluorescens strain WGS365 was a gift of Dr. Cara Haney, University of British Columbia. E. faecalis OG1RF was a gift of Dr. Danielle Garsin, University of Texas, McGovern Medical School.

\section{SUPPLEMENTARY MATERIAL}

The Supplementary Material for this article can be found online at: https://www.frontiersin.org/articles/10.3389/fmicb. 2018.03317/full\#supplementary-material

therapeutic agent. Proc. Natl. Acad. Sci. U.S.A 105, 16761-16766. doi: 10.1073/ pnas.0808608105

Banin, E., Vasil, M. L., and Greenberg, E. P. (2005). Iron and Pseudomonas aeruginosa biofilm formation. Proc. Natl. Acad. Sci. U.S.A. 102, 11076-11081. doi: $10.1073 /$ pnas. 0504266102

Beanan, M. J., and Strome, S. (1992). Characterization of a germ-line proliferation mutation in C. elegans. Development 116, 755-766.

Beare, P. A., For, R. J., Martin, L. W., and Lamont, I. L. (2003). Siderophoremediated cell signalling in Pseudomonas aeruginosa: divergent pathways regulate virulence factor production and siderophore receptor synthesis. Mol. Microbiol. 47, 195-207. doi: 10.1046/j.1365-2958.2003.03288.x

Bonchi, C., Frangipani, E., Imperi, F., and Visca, P. (2015). Pyoverdine and proteases affect the response of Pseudomonas aeruginosa to gallium in human serum. Antimicrob. Agents Chemother. 59, 5641-5646. doi: 10.1128/AAC. 01097-15

Cezairliyan, B., Vinayavekhin, N., Grenfell-Lee, D., Yuen, G. J., Saghatelian, A., and Ausubel, F. M. (2013). Identification of Pseudomonas aeruginosa phenazines that kill caenorhabditis elegans. PLoS Pathog. 9:e1003101. doi: 10.1371/journal. ppat.1003101

Cheng, G., Sa, W., Cao, C., Guo, L., Hao, H., Liu, Z., et al. (2016). Quinoxaline 1,4di-N-Oxides: biological activities and mechanisms of actions. Front. Pharmacol. 7:64. doi: 10.3389/fphar.2016.00064 
Clevenger, K. D., Wu, R., Er, J. A., Liu, D., and Fast, W. (2013). Rational design of a transition state analogue with picomolar affinity for Pseudomonas aeruginosa PvdQ, a siderophore biosynthetic enzyme. ACS Chemical Biol. 8, 2192-2200. doi: $10.1021 / \mathrm{cb} 400345 \mathrm{~h}$

Congreve, M., Carr, R., Murray, C., and Jhoti, H. (2003). A 'rule of three' for fragment-based lead discovery? Drug Discov. Today 8, 876-877. doi: 10.1016/ S1359-6446(03)02831-9

Costabile, G., d'Angelo, I., d'Emmanuele, di Villa, Bianca, R., Mitidieri, E., et al. (2016). Development of inhalable hyaluronan/mannitol composite dry powders for flucytosine repositioning in local therapy of lung infections. J. Control Release 238, 80-91. doi: 10.1016/j.jconrel.2016.07.029

Cushnie, T. P., O'Driscoll, N. H., and Lamb, A. J. (2016). Morphological and ultrastructural changes in bacterial cells as an indicator of antibacterial mechanism of action. Cell. Mol. Life Sci. 73, 4471-4492. doi: 10.1007/s00018016-2302-2

D'Angelo, F., Baldelli, V., Halliday, N., Pantalone, P., Polticelli, F., Fiscarelli, E., et al. (2018). Identification of FDA-approved drugs as antivirulence agents targeting the pqs quorum-sensing system of Pseudomonas aeruginosa. Antimicrob. Agents Chemother. 62:e1296-18. doi: 10.1128/AAC.01296-18

De Vos, D., De Chial, M., Cochez, C., Jansen, S., Tummler, B., Meyer, J. M., et al. (2001). Study of pyoverdine type and production by Pseudomonas aeruginosa isolated from cystic fibrosis patients: prevalence of type II pyoverdine isolates and accumulation of pyoverdine-negative mutations. Arch. Microbiol. 175, 384-388. doi: 10.1007/s002030100278

DeLeon, K., Balldin, F., Watters, C., Hamood, A., Griswold, J., Sreedharan, S., et al. (2009). Gallium maltolate treatment eradicates Pseudomonas aeruginosa infection in thermally injured mice. Antimicrob. Agents Chemother. 53, 13311337. doi: 10.1128/AAC.01330-08

Doak, B. C., Norton, R. S., and Scanlon, M. J. (2016). The ways and means of fragment-based drug design. Pharmacol. Ther. 167, 28-37. doi: 10.1016/j. pharmthera.2016.07.003

Dortet, L., Lombardi, C., Cretin, F., Dessen, A., and Filloux, A. (2018). Poreforming activity of the Pseudomonas aeruginosa type III secretion system translocon alters the host epigenome. Nat. Microbiol. 3, 378-386. doi: 10.1038/ s41564-018-0109-7

Drake, E. J., and Gulick, A. M. (2011). Structural characterization and highthroughput screening of inhibitors of PvdQ, an NTN hydrolase involved in pyoverdine synthesis. ACS Chem. Biol. 6, 1277-1286. doi: 10.1021/cb2002973

Fuchs, R., Schafer, M., Geoffroy, V., and Meyer, J. M. (2001). Siderotyping-a powerful tool for the characterization of pyoverdines. Curr. Top. Med. Chem. 1, 31-57. doi: 10.2174/1568026013395542

Garcia-Contreras, R., Perez-Eretza, B., Lira-Silva, E., Jasso-Chavez, R., CoriaJimenez, R., Rangel-Vega, A., et al. (2014). Gallium induces the production of virulence factors in Pseudomonas aeruginosa. Pathog. Dis. 70, 95-98. doi: 10.1111/2049-632X.12105

Garsin, D. A., Sifri, C. D., Mylonakis, E., Qin, X., Singh, K. V., Murray, B. E., et al. (2001). A simple model host for identifying Gram-positive virulence factors. Proc. Natl. Acad. Sci. U.S.A. 98, 10892-10897. doi: 10.1073/pnas.19137 8698

Geels, F. P., and Schippers, B. (1983). Reduction of yield depressions in high frequency potato cropping soil after seed tuber treatments with antagonistic fluorescent Pseudomonas spp. J. Phytopathol. 108, 207-214. doi: 10.1111/j.14390434.1983.tb00580.x

Greenwald, J., Hoegy, F., Nader, M., Journet, L., Mislin, G. L., Graumann, P. L., et al. (2007). Real time fluorescent resonance energy transfer visualization of ferric pyoverdine uptake in Pseudomonas aeruginosa. A role for ferrous iron. J. Biol. Chem. 282, 2987-2995. doi: 10.1074/jbc.M60923 8200

Holloway, B. W., Romling, U., and Tummler, B. (1994). Genomic mapping of Pseudomonas aeruginosa PAO. Microbiology 140( Pt 11), 2907-2929. doi: 10. 1099/13500872-140-11-2907

Imperi, F., Leoni, L., and Visca, P. (2014). Antivirulence activity of azithromycin in Pseudomonas aeruginosa. Front. Microbiol. 5:178. doi: 10.3389/fmicb.2014. 00178

Imperi, F., Massai, F., Facchini, M., Frangipani, E., Visaggio, D., Leoni, L., et al. (2013). Repurposing the antimycotic drug flucytosine for suppression of Pseudomonas aeruginosa pathogenicity. Proc. Natl. Acad. Sci. U.S.A. 110, 7458-7463. doi: 10.1073/pnas. 1222706110
Imperi, F., Tiburzi, F., and Visca, P. (2009). Molecular basis of pyoverdine siderophore recycling in Pseudomonas aeruginosa. Proc. Natl. Acad. Sci. U.S.A. 106, 20440-20445. doi: 10.1073/pnas.0908760106

Ismail, M. M., Amin, K. M., Noaman, E., Soliman, D. H., and Ammar, Y. A. (2010). New quinoxaline 1, 4-di-N-oxides: anticancer and hypoxia-selective therapeutic agents. Eur. J. Med. Chem. 45, 2733-2738. doi: 10.1016/j.ejmech.2010.02.052

Kaneko, Y., Thoendel, M., Olakanmi, O., Britigan, B. E., and Singh, P. K. (2007). The transition metal gallium disrupts Pseudomonas aeruginosa iron metabolism and has antimicrobial and antibiofilm activity. J. Clin. Invest. 117, 877-888. doi: 10.1172/JCI30783

Kang, D., Kirienko, D. R., Webster, P., Fisher, A. L., and Kirienko, N. V. (2018). Pyoverdine, a siderophore from Pseudomonas aeruginosa, translocates into C. elegans, removes iron, and activates a distinct host response. Virulence 9, 804-817. doi: 10.1080/21505594.2018.1449508

Kang, D., and Kirienko, N. V. (2017). High-Throughput genetic screen reveals that early attachment and biofilm formation are necessary for full pyoverdine production by Pseudomonas aeruginosa. Front. Microbiol. 8:1707. doi: 10.3389/ fmicb.2017.01707

Kang, D., and Kirienko, N. V. (2018). Interdependence between iron acquisition and biofilm formation in Pseudomonas aeruginosa. J. Microbiol. 56, 449-457. doi: 10.1007/s12275-018-8114-3

Kirienko, D. R., Revtovich, A. V., and Kirienko, N. V. (2016). A high-content, phenotypic screen identifies fluorouridine as an inhibitor of pyoverdine biosynthesis and Pseudomonas aeruginosa virulence. mSpher 1:e217-16. doi: 10.1128/mSphere.00217-16

Kirienko, N. V., Ausubel, F. M., and Ruvkun, G. (2015). Mitophagy confers resistance to siderophore-mediated killing by Pseudomonas aeruginosa. Proc. Natl. Acad. Sci. U.S.A. 112, 1821-1826. doi: 10.1073/pnas.1424954112

Kirienko, N. V., Cezairliyan, B. O., Ausubel, F. M., and Powell, J. R. (2014). Pseudomonas aeruginosa PA14 pathogenesis in caenorhabditis elegans. Methods Mol. Biol. 1149, 653-669. doi: 10.1007/978-1-4939-0473-0_50

Kirienko, N. V., Kirienko, D. R., Larkins-Ford, J., Wählby, C., Ruvkun, G., and Ausubel, F. M. (2013). Pseudomonas aeruginosa disrupts caenorhabditis elegans iron homeostasis, causing a hypoxic response and death. Cell Host Microbe 13, 406-416. doi: 10.1016/j.chom.2013.03.003

Kirienko, N. V., McEnerney, J. D., and Fay, D. S. (2008). Coordinated regulation of intestinal functions in C. elegans by LIN-35/Rb and SLR-2. PLoS Genet. 4:e1000059. doi: 10.1371/journal.pgen.1000059

Köhler, T., Michéa-Hamzehpour, M., Henze, U., Gotoh, N., Curty, L. K., and Pechère, J. C. (1997). Characterization of MexE-MexF-OprN, a positively regulated multidrug efflux system of Pseudomonas aeruginosa. Mol. Microbiol. 23, 345-354. doi: 10.1046/j.1365-2958.1997.2281594.x

Kon, K., and Rai, M. (2016). Antibiotic Resistance: Mechanisms and New Antimicrobila Approaches. Cambridge: Academic Press.

Kuchma, S. L., Brothers, K. M., Merritt, J. H., Liberati, N. T., Ausubel, F. M., and O'Toole, G. A. (2007). BifA, a cyclic-Di-GMP phosphodiesterase, inversely regulates biofilm formation and swarming motility by Pseudomonas aeruginosa PA14. J. Bacteriol. 189, 8165-8178. doi: 10.1128/JB.00586-07

Lamont, I. L., Beare, P. A., Ochsner, U., Vasil, A. I., and Vasil, M. L. (2002). Siderophore-mediated signaling regulates virulence factor production in Pseudomonas aeruginosa. Proc. Natl. Acad. Sci. U.S.A 99, 7072-7077. doi: 10. 1073/pnas.092016999

Liberati, N. T., Urbach, J. M., Miyata, S., Lee, D. G., Drenkard, E., Wu, G., et al. (2006). An ordered, nonredundant library of Pseudomonas aeruginosa strain PA14 transposon insertion mutants. Proc. Natl. Acad. Sci. U.S.A. 103, 2833-2838. doi: 10.1073/pnas.0511100103

Lipinski, C. A., Lombardo, F., Dominy, B. W., and Feeney, P. J. (2001). Experimental and computational approaches to estimate solubility and permeability in drug discovery and development settings. Adv. Drug Deliv. Rev. 46, 3-26. doi: 10.1016/S0169-409X(00)00129-0

Liu, P. V., Matsumoto, H., Kusama, H., and Bergan, T. (1983). Survey of heatstable, major somatic antigens of Pseudomonas aeruginosa. Int. J. Syst. Bacteriol. 33, 256-264. doi: 10.1099/00207713-33-2-256

Lomovskaya, O., Warren, M. S., Lee, A., Galazzo, J., Fronko, R., Lee, M., et al. (2001). Identification and characterization of inhibitors of multidrug resistance efflux pumps in Pseudomonas aeruginosa: novel agents for combination therapy. Antimicrob. Agents Chemother. 45, 105-116. doi: 10.1128/AAC.45.1. 105-116.2001 
Lopez-Medina, E., Fan, D., Coughlin, L. A., Ho, E. X., Lamont, I. L., Reimmann, C., et al. (2015). Candida albicans inhibits Pseudomonas aeruginosa virulence through suppression of pyochelin and pyoverdine biosynthesis. PLoS Pathog. 11:e1005129. doi: 10.1371/journal.ppat.1005129

Mah, T. F., and O’Toole, G. A. (2001). Mechanisms of biofilm resistance to antimicrobial agents. Trends Microbiol. 9, 34-39. doi: 10.1016/S0966-842X(00) 01913-2

Managò, A., Becker, K. A., Carpinteiro, A., Wilker, B., Soddemann, M., Seitz, A. P., et al. (2015). Pseudomonas aeruginosa pyocyanin induces neutrophil death via mitochondrial reactive oxygen species and mitochondrial acid sphingomyelinase. Antioxid. Redox Signal. 22, 1097-1110. doi: 10.1089/ars. 2014.5979

Medeiros, A. A., O’Brien, T. F., Wacker, W. E., and Yulug, N. F. (1971). Effect of salt concentration on the apparent in-vitro susceptibility of Pseudomonas and other gram-negative bacilli to gentamicin. J. Infect. Dis. 124(Suppl.), S59-S64. doi: 10.1093/infdis/124.Supplement_1.S59

Meyer, J. M., Geoffroy, V. A., Baida, N., Gardan, L., Izard, D., Lemanceau, P., et al. (2002). Siderophore typing, a powerful tool for the identification of fluorescent and nonfluorescent pseudomonads. Appl. Environ. Microbiol. 68, 2745-2753. doi: 10.1128/AEM.68.6.2745-2753.2002

Meyer, J. M., Neely, A., Stintzi, A., Georges, C., and Holder, I. A. (1996). Pyoverdin is essential for virulence of Pseudomonas aeruginosa. Infect. Immun. $64,518-523$.

Meyer, J. M., Stintzi, A., Coulanges, V., Shivaji, S., Voss, J. A., Taraz, K., et al. (1998). Siderotyping of fluorescent pseudomonads: characterization of pyoverdines of Pseudomonas fluorescens and Pseudomonas putida strains from Antarctica. Microbiology 144(Pt 11), 3119-3126.

Meyer, J. M., Stintzi, A., De Vos, D., Cornelis, P., Tappe, R., Taraz, K., et al. (1997). Use of siderophores to type pseudomonads: the three Pseudomonas aeruginosa pyoverdine systems. Microbiology 143( Pt 1), 35-43. doi: 10.1099/00221287143-1-35

Minandri, F., Bonchi, C., Frangipani, E., Imperi, F., and Visca, P. (2014). Promises and failures of gallium as an antibacterial agent. Future Microbiol. 9, 379-397. doi: $10.2217 /$ fmb.14.3

Minandri, F., Imperi, F., Frangipani, E., Bonchi, C., Visaggio, D., Facchini, M., et al. (2016). Role of iron uptake systems in Pseudomonas aeruginosa virulence and airway infection. Infect. Immun. 84, 2324-2335. doi: 10.1128/IAI.00098-16

Murray, C. W., and Rees, D. C. (2009). The rise of fragment-based drug discovery. Nat. Chem. 1, 187-192. doi: 10.1038/nchem.217

Nadal Jimenez, P., Koch, G., Papaioannou, E., Wahjudi, M., Krzeslak, J., Coenye, T., et al. (2010). Role of PvdQ in Pseudomonas aeruginosa virulence under ironlimiting conditions. Microbiology 156, 49-59. doi: 10.1099/mic.0.030973-0

Ochsner, U. A., Johnson, Z., Lamont, I. L., Cunliffe, H. E., and Vasil, M. L. (1996). Exotoxin a production in Pseudomonas aeruginosa requires the iron-regulated pvdS gene encoding an alternative sigma factor. Mol. Microbiol. 21, 1019-1028. doi: 10.1046/j.1365-2958.1996.481425.x

Paczkowski, J. E., Mukherjee, S., McCready, A. R., Cong, J. P., Aquino, C. J., Kim, H., et al. (2017). Flavonoids suppress Pseudomonas aeruginosa virulence through allosteric inhibition of quorum-sensing receptors. J. Biol. Chem. 292, 4064-4076. doi: 10.1074/jbc.M116.770552

Poole, K., Gotoh, N., Tsujimoto, H., Zhao, Q., Wada, A., Yamasaki, T., et al. (1996). Overexpression of the mexC-mexD-oprJ efflux operon in nfxB-type multidrugresistant strains of Pseudomonas aeruginosa. Mol. Microbiol. 21, 713-724. doi: 10.1046/j.1365-2958.1996.281397.x

Poole, K., Krebes, K., McNally, C., and Neshat, S. (1993). Multiple antibiotic resistance in Pseudomonas aeruginosa: evidence for involvement of an efflux operon. J. Bacteriol. 175, 7363-7372. doi: 10.1128/jb.175.22.7363-7372.1993

Pukkila-Worley, R., Feinbaum, R., Kirienko, N. V., Larkins-Ford, J., Conery, A. L., and Ausubel, F. M. (2012). Stimulation of host immune defenses by a small molecule protects C. elegans from bacterial infection. PLoS Genet. 8:e1002733. doi: 10.1371/journal.pgen.1002733

Rahme, L. G., Stevens, E. J., Wolfort, S. F., Shao, J., Tompkins, R. G., and Ausubel, F. M. (1995). Common virulence factors for bacterial pathogenicity in plants and animals. Science 268, 1899-1902. doi: 10.1126/science.7604262

Rezzoagli, C., Wilson, D., Weigert, M., Wyder, S., and Kummerli, R. (2018). Probing the evolutionary robustness of two repurposed drugs targeting iron uptake in Pseudomonas aeruginosa. Evol. Med. Public Health 2018, 246-259. doi: 10.1093/emph/eoy026

Saliba, A. M., Filloux, A., Ball, G., Silva, A. S., Assis, M. C., and Plotkowski, M. C. (2002). Type III secretion-mediated killing of endothelial cells by Pseudomonas aeruginosa. Microb. Pathog. 33, 153-166. doi: 10.1016/S0882-4010(02)90522-X

Shanks, R. M., Caiazza, N. C., Hinsa, S. M., Toutain, C. M., and O’Toole, G. A. (2006). Saccharomyces cerevisiae-based molecular tool kit for manipulation of genes from gram-negative bacteria. Appl. Environ. Microbiol. 72, 5027-5036. doi: 10.1128/AEM.00682-06

Stiernagle, T. (2006). Maintenance of C. elegans. WormBook: the online review of C elegans biology. Minneapolis, MN: University of Minnesota.

Suzuki, H., Pangborn, J., and Kilgore, W. W. (1967). Filamentous cells of Escherichia coli formed in the presence of mitomycin. J. Bacteriol. 93, 683-688.

Takase, H., Nitanai, H., Hoshino, K., and Otani, T. (2000). Impact of siderophore production on Pseudomonas aeruginosa infections in immunosuppressed mice. Infect. Immun. 68, 1834-1839. doi: 10.1128/IAI.68.4.1834-1839.2000

Tan, S. Y., Chua, S. L., Chen, Y., Rice, S. A., Kjelleberg, S., Nielsen, T. E., et al. (2013). Identification of five structurally unrelated quorum-sensing inhibitors of Pseudomonas aeruginosa from a natural-derivative database. Antimicrob. Agents Chemother. 57, 5629-5641. doi: 10.1128/AAC.00955-13

Theriault, J. R., Wurst, J., Jewett, I., Verplank, L., Perez, J. R., Gulick, A. M., et al. (2010). "Identification of a small molecule inhibitor of Pseudomonas aeruginosa PvdQ acylase, an enzyme involved in siderophore pyoverdine synthesis," in Probe Reports from the NIH Molecular Libraries Program, (Bethesda MD: National Center for Biotchnology Information).

Tjahjono, E., and Kirienko, N. V. (2017). A conserved mitochondrial surveillance pathway is required for defense against Pseudomonas aeruginosa. PLoS Genet. 13:e1006876. doi: 10.1371/journal.pgen.1006876

van Tilburg Bernardes, E., Charron-Mazenod, L., Reading, D. J., ReckseidlerZenteno, S. L., and Lewenza, S. (2017). Exopolysaccharide-repressing small molecules with antibiofilm and antivirulence activity against Pseudomonas aeruginosa. Antimicrob. Agents Chemother. 61:e1997-16. doi: 10.1128/AAC. 01997-16

Vandeputte, O. M., Kiendrebeogo, M., Rasamiravaka, T., Stévigny, C., Duez, P., Rajaonson, S., et al. (2011). The flavanone naringenin reduces the production of quorum sensing-controlled virulence factors in Pseudomonas aeruginosa PAO1. Microbiology 157, 2120-2132. doi: 10.1099/mic.0.049338-0

Walker, J. R., and Pardee, A. B. (1968). Evidence for a relationship between deoxyribonucleic acid metabolism and septum formation in Escherichia coli. J. Bacteriol. 95, 123-131.

Wilderman, P. J., Vasil, A. I., Johnson, Z., Wilson, M. J., Cunliffe, H. E., Lamont, I. L., et al. (2001). Characterization of an endoprotease (PrpL) encoded by a PvdS-regulated gene in Pseudomonas aeruginosa. Infect. Immun. 69, 5385-5394. doi: 10.1128/IAI.69.9.5385-5394.2001

Wurst, J. M., Drake, E. J., Theriault, J. R., Jewett, I. T., VerPlank, L., Perez, J. R., et al. (2014). Identification of inhibitors of PvdQ, an enzyme involved in the synthesis of the siderophore pyoverdine. ACS Chem. Biol. 9, 1536-1544. doi: $10.1021 / \mathrm{cb} 5001586$

Xiao, R., and Kisaalita, W. S. (1997). Iron acquisition from transferrin and lactoferrin by Pseudomonas aeruginosa pyoverdin. Microbiology 143(Pt 7), 2509-2515. doi: 10.1099/00221287-143-7-2509

Yabuuchi, E., and Ohyama, A. (1972). Characterization of "Pyomelanin"Producing Strains of Pseudomonas aeruginosa. Int. J. Syst. Bacteriol. 22, 53-64. doi: 10.1099/00207713-22-2-53

Conflict of Interest Statement: The authors declare that the research was conducted in the absence of any commercial or financial relationships that could be construed as a potential conflict of interest.

Copyright (c) 2019 Kirienko, Kang and Kirienko. This is an open-access article distributed under the terms of the Creative Commons Attribution License (CC BY). The use, distribution or reproduction in other forums is permitted, provided the original author(s) and the copyright owner(s) are credited and that the original publication in this journal is cited, in accordance with accepted academic practice. No use, distribution or reproduction is permitted which does not comply with these terms. 